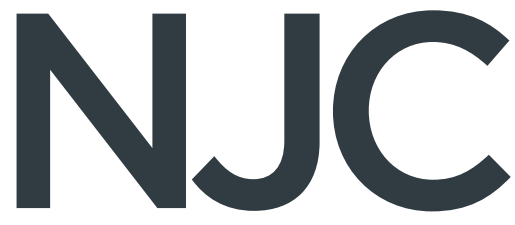

New Journal of Chemistry

A journal for new directions in chemistry

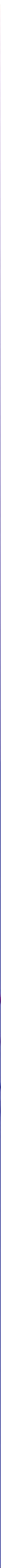

ISSN 1144-0546

\title{
PAPER
}

Rashid IImi, Paul R. Raithby, Muhammad S. Khan et al.

Towards white light emission from a hybrid thin film of a self-assembled ternary samarium(III) complex

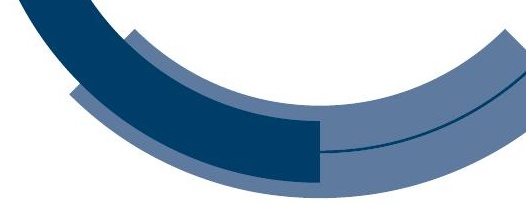


Check for updates

Cite this: New J. Chem., 2020, 44,5673

Received 19th December 2019, Accepted 7th February 2020

DOI: 10.1039/c9nj06287d

rsc.li/njc

\section{Towards white light emission from a hybrid thin film of a self-assembled ternary samarium(III) complex $\dagger$}

\author{
Rashid IImi, (D)*a Sevgi Kansız, (D) ${ }^{b}$ Nawal K. Al-Rasbi, ${ }^{a}$ Necmi Dege, ${ }^{c}$ \\ Paul R. Raithby iD *d and Muhammad S. Khan (iD *a
}

\begin{abstract}
A new samarium complex $\left[\mathrm{Sm}(\mathrm{hfaa})_{3}(\mathrm{Py}-\mathrm{Im})\right](\mathrm{ML})$ was synthesized by utilizing hexafluoroacetylacetone (hfaa) and 2-(2-pyridyl)benzimidazole (Py- $\mathrm{Im}$ ) as coordinating ligands. Single crystal X-ray analysis shows that in the solid-state the complex dimerizes through intermolecular hydrogen-bonding with $\mathrm{N}-\mathrm{H} \ldots \mathrm{O}$ $(2.127 \AA)$ and $\mathrm{N}-\mathrm{H} \ldots \mathrm{F}(2.576 \AA)$ interactions. The complex in the solid-state displayed highly monochromatic brilliant red emission with Commission International de I'Eclairage (CIE) color coordinates of 0.6532; 0.3336 , with a remarkably long luminescence lifetime $\left(\tau_{\exp .} \approx 204.47 \pm 4.043 \mu \mathrm{s}\right)$ and a high intrinsic quantum yield $\left(Q_{S m}^{S m} \approx 6.60 \%\right)$. Furthermore, the complex in different organic media displayed color tunability from orange

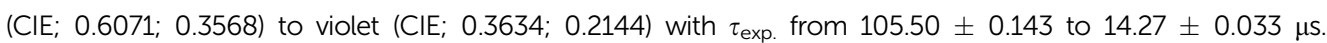
Hybrid and flexible thin films obtained by doping different concentrations (1\%, $2 \%, 4 \%$ and $6 \%$ ) of the complex into the poly(urethane) (PU) polymer matrix also exhibited color tunability from violet (CIE; 0.3380 ; 0.2162 ) to light orange ( $\mathrm{CIE} ; 0.5666 ; 0.3408)$. White light emission from a $1 \%$ doped thin film of the complex was realized by changing the excitation wavelength. Thus, the new material could be a potential candidate for the fabrication of full-color display devices and for solid-state lighting (SSL) applications.
\end{abstract}

\section{Introduction}

Highly photoluminescent lanthanide (Ln) complexes incorporating organic ligands that utilize the antenna effect to generate efficient luminescent materials continue to be of interest to academics and industrialists because of their applications. A range of intriguing technological applications incorporating organo-lanthanide complexes has been developed such as organic light emitting diodes (OLEDs), ${ }^{1}$ laser materials, ${ }^{2}$ sensitizers to improve the electroluminescence (EL) of red and green emitting iridium complexes, ${ }^{1,3}$ luminescent thermometers, ${ }^{4}$ or sensors. ${ }^{5}$ The extensive research efforts are due to the unique optical properties of trivalent lanthanide ions [Ln(III)], which feature fingerprint emissions for each $\operatorname{Ln}(\mathrm{III})$ ion spanning through the visible to near-infrared (NIR) region of the spectrum, exhibit highly

\footnotetext{
${ }^{a}$ Department of Chemistry, Sultan Qaboos University, P.O. Box 36, Al Khod 123, Oman.E-mail: rashidilmi@gmail.com,msk.squ.edu@gmail.com,msk@squ.edu.om

${ }^{b}$ Department of Fundamental Sciences, Faculty of Engineering, Samsun University, 55420, Samsun, Turkey

${ }^{c}$ Department of Physics, Faculty of Arts and Sciences, Ondokuz Mayıs University, 55139, Samsun, Turkey

${ }^{d}$ Department of Chemistry, University of Bath, Claverton Down, Bath BA2 7AY, UK. E-mail: p.r.raithby@bath.ac.uk

$\dagger$ Electronic supplementary information (ESI) available. CCDC 1943973. For ESI and crystallographic data in CIF or other electronic format see DOI: 10.1039/c9nj06287d
}

monochromatic emissions, and have long-lived excited lifetimes. However, most of the work reported to date deals with either $\mathrm{Eu}(\mathrm{III})$ or $\mathrm{Tb}$ (III) that emits intense red and green light. Work related to luminescent $\mathrm{Sm}$ (III) complexes is very limited by comparison.

Generally, an efficient Sm(III) complex would display a deep red emission ${ }^{6}$ because of the presence of the electric-dipole ${ }^{4} \mathrm{G}_{5 / 2} \rightarrow{ }^{6} \mathrm{H}_{9 / 2}$ transition at $648 \mathrm{~nm}$ and thus is beneficial for constructing the red element in a full-color display. Moreover, the emission spectrum of Sm(III) ion displays two more medium intensity transitions at $563 \mathrm{~nm}$ (greenish-yellow emission) and $606 \mathrm{~nm}$ (yellowish-orange emission). The presence of these two medium intensity emission transitions in the spectrum offers emission color tunability; if the emission is not very efficient that would lead to residual ligand fluorescence (RLF) in the blue region of the spectrum. In fact, proper amalgamation of these colors in Sm(III) complex(es) could result in white light emission from a single molecule platform. ${ }^{7}$ This is achievable by change in concentration, excitation, temperature and solvent medium or by embedding the complex into a polymer matrix. ${ }^{7 b, d, e}$ Motivated by these ideas, and following on from some previous luminescence studies of Sm(III) hexafluoroacetylacetone complexes, ${ }^{8}$ we have synthesized a new ternary Sm(III) complex using hexafluoroacetylacetone (hfaa) as the primary antenna and 2-(2pyridyl)benzimidazole (Py-Im) as the secondary ligand (Scheme 1). The primary hfaa ligand was chosen because of the presence of 


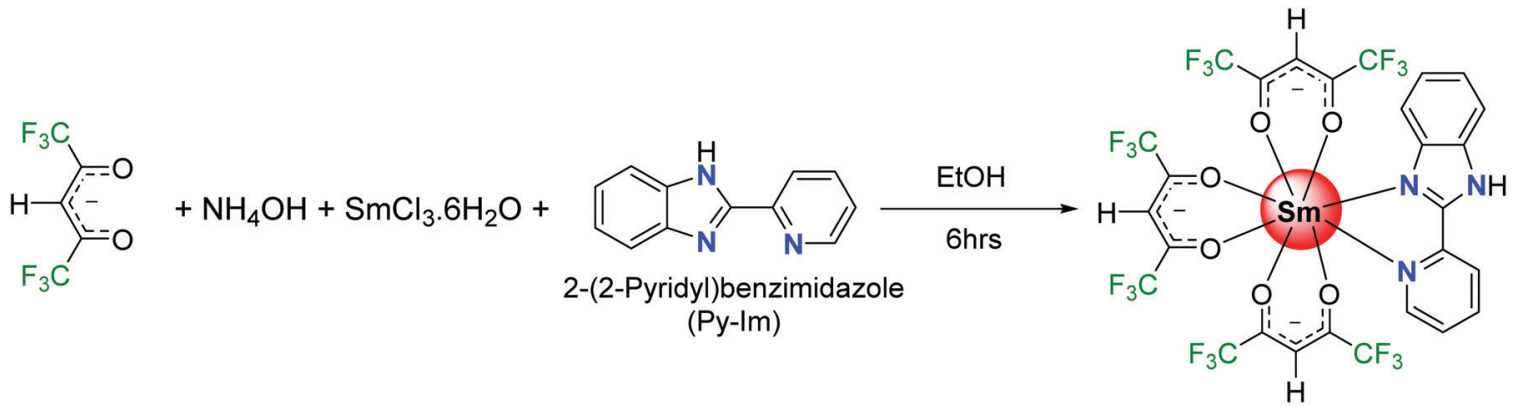

Scheme 1 Synthesis of $\left[\mathrm{Sm}(\mathrm{hfaa})_{3}(\mathrm{Py}-\mathrm{Im})\right]$ complex.

low vibrational bonds (C-F) that suppress radiationless transitions via vibrational relaxations. ${ }^{9}$ The bidentate secondary Py-Im ligand was chosen because of its compatible triplet state $\left({ }^{3} \pi \pi^{*} \approx 20576 \mathrm{~cm}^{-1}\right)^{1 a}$ and its asymmetrical $N, N$-chelating coordination mode via the five-membered imidazolyl nitrogen and the six-membered pyridyl nitrogen atoms, and thus could be useful in enhancing the radiative decay rate. ${ }^{10}$ Moreover, the $\mathrm{NH}$ proton of the imidazolyl ring is usually involved in hydrogen bond formation and self-assembly, resulting in fascinating structures and arrangements in the solid-state. The complex was characterized by analytical and spectroscopic methods and the solid-state structure was determined by single crystal X-ray diffraction (SC-XRD). It was found that $\mathrm{NH}$ proton in the complex contributes to the formation of a hydrogen bonded dimer in the solid-state, which is not common in this class of complexes. ${ }^{11}$ The photophysical properties of the complex were analyzed in detail by steady-state excitation and emission spectroscopy as well as by time-resolved spectroscopy in the solid-state and in different organic media (dichloromethane, acetonitrile, acetone, tetrahydrofuran, ethanol and methanol). The complex exhibits color tunability and shows a brilliant red emission in the solid-state with a remarkably long luminescence lifetime $\left(\tau_{\exp .} \approx 204.47 \pm 4.043 \mu \mathrm{s}\right)$ and an intrinsic quantum yield of $Q_{\mathrm{Sm}}^{\mathrm{Sm}} \approx 6.60 \%$. Upon changing the medium, the complex displays color tunability from orange to violet with an obvious change in the luminescence lifetime, thus hinting towards white light generation. Considering these interesting photophysical properties and the possibility of generating white light emission at a single component level, the complex was further embedded into a PU polymer matrix with different doping concentrations to develop color-tunable flexible hybrid thin films with a view to developing high-performance luminescent materials for optoelectronic application.

\section{Experimental section}

\subsection{Materials, syntheses and analyses}

$\mathrm{SmCl}_{3} \cdot 6 \mathrm{H}_{2} \mathrm{O}$ was purchased from Strem Chemicals, Inc. USA, while other chemicals were obtained from Sigma Aldrich and used as received. Solvents were pre-dried and distilled before use according to standard procedures. ${ }^{12}$ Elemental analysis was performed on Euro EA - CHN Elemental Analyser in the Department of Chemistry, Sultan Qaboos University. Attenuated total-reflectance
(ATR) infrared (IR) spectra of pure solid samples were recorded on diamond using a Cary 630 FT-IR spectrometer. Mass spectra were obtained using a VG Autospec magnetic sector instrument using electrospray ionization (ESI).

\subsection{Synthesis of the $\left[\mathrm{Sm}(\mathrm{hfaa})_{3}(\mathrm{Py}-\mathrm{Im})\right]$ ternary complex}

The samarium complex was synthesized by a one-step reaction at room temperature (RT) (Scheme 1). To an ethanolic hfaa $(0.73 \mathrm{~g} ; 3.50 \mathrm{mmol})$ solution, $25 \%$ ethanolic ammonia $(0.27 \mathrm{~mL})$ solution was added dropwise with constant stirring. After the mixture was stirred for a further $30 \mathrm{~min}$, ethanolic solutions of Py-Im $(0.226 \mathrm{~g}, 1.16 \mathrm{mmol})$ and $\mathrm{SmCl}_{3} \cdot 6 \mathrm{H}_{2} \mathrm{O}(0.422 \mathrm{~g}, 1.16 \mathrm{mmol})$ were added sequentially, and the reaction mixture was left with stirring for a further $6 \mathrm{~h}$. The solution was left to allow slow solvent evaporation. After a week, a yellow powder precipitated. The precipitate was filtered, washed with ice-cold ethanol followed by hexane, and dried in the air to obtain a yellow solid in $65 \%$ yield. Calculated for $\mathrm{C}_{27} \mathrm{H}_{12} \mathrm{~F}_{18} \mathrm{~N}_{3} \mathrm{O}_{6} \mathrm{Sm}$ : C, $33.55 ; \mathrm{H}, 1.25 ; \mathrm{N}$, 4.35; found $\mathrm{C}, 33.88 ; \mathrm{H}, 1.20 ; \mathrm{N}, 4.29 \%$. ESI-MS - $\left[\mathrm{M}+\mathrm{K}-\left(\mathrm{CH}_{3}\right)_{2} \mathrm{CO}\right]$; $\mathrm{m} / \mathrm{z}=948.1$; melting point $\left(T_{\mathrm{m}}\right)=195^{\circ} \mathrm{C}$.

\subsection{Single crystal X-ray diffraction analyses}

A single crystal of $\left[\mathrm{Sm}(\mathrm{hfaa})_{3}(\mathrm{Py}-\mathrm{Im})\right]$ with dimensions of $0.77 \times$ $0.57 \times 0.43 \mathrm{~mm}$ was selected for X-ray diffraction. The intensity data was collected at $296 \mathrm{~K}$ on a STOE IPDS 2 diffractometer equipped with graphite monochromatic Mo-K $\alpha$ radiation $(\lambda=$ $0.71073 \AA$ Å). The structure was solved by direct methods using SHELXT $^{13}$ and refined by full-matrix least-squares methods on $F^{2}$ using SHELXL17/1. ${ }^{14}$ All the hydrogen atoms were placed in calculated positions and refined using a riding model, with $\mathrm{C}-\mathrm{H}=0.93 \AA$ for $\mathrm{CH} \mathrm{H}$-atoms, and $U_{\text {iso }}(\mathrm{H})=1.2 U_{\text {eq }}(\mathrm{C})$. The $\mathrm{N}$-bound $\mathrm{H}$-atom was positioned geometrically $(\mathrm{N}-\mathrm{H}=0.86 \AA)$ and refined with a riding model, with $U_{\text {iso }}(\mathrm{H})=1.2 U_{\text {eq }}(\mathrm{N})$. The molecular geometry calculations and drawings were performed with Mercury for Windows ${ }^{15}$ and PLATON. ${ }^{16}$ WinGX $^{17}$ was used to prepare material for publication. The crystal data, data collection and refinement parameters for $\left[\mathrm{Sm}(\mathrm{hfaa})_{3}(\mathrm{Py}-\mathrm{Im})\right]$ are presented in Table S1, CCDC 1943973.†

\subsection{Hirshfeld surface analysis}

Hirshfeld surface (HS) analysis (including fingerprint plots) was used to understand the interactions involved in the crystal packing. The Hirshfeld surface analysis ${ }^{18}$ and the associated 
two-dimensional fingerprint plots ${ }^{19}$ were generated using the CrystalExplorer17 package. $^{20}$ The normalized contact distance ( $\left.d_{\text {norm }}\right)$ was calculated via the following expression:

$$
d_{\text {norm }}=\frac{d_{\mathrm{i}}-r_{\mathrm{i}}^{\mathrm{vdW}}}{r_{\mathrm{i}}^{\mathrm{vdW}}}+\frac{d_{\mathrm{e}}-r_{\mathrm{e}}^{\mathrm{vdW}}}{r_{\mathrm{e}}^{\mathrm{vdW}}}
$$

$d_{\mathrm{e}}$ and $d_{i}$ are distances from a point on the surface to the nearest nucleus outside the HS and to the nearest nucleus inside the HS, respectively. $r_{\mathrm{i}}^{\mathrm{vdw}}$ and $r_{\mathrm{e}}^{\mathrm{vdw}}$ terms are the van der Waals radii of the atom, which lie inside the HS and outside of the HS, respectively. We employed the final validated CIF as the input to CrystalExplorer17 to conduct the above calculations for the samarium complex.

\subsection{Hybrid thin film fabrication of $\left[\operatorname{Sm}(h f a a)_{3}(\operatorname{Py}-\operatorname{Im})\right] @ P U \_x \%$}

A PU composite thin film of the $\left[\mathrm{Sm}(\mathrm{hfaa})_{3}(\mathrm{Py}-\mathrm{Im})\right]$ complex was fabricated by dissolving PU $(4 \% \mathrm{w} / \mathrm{v})$ in tetrahydrofuran (THF) $(25 \mathrm{~mL})$ The suspension was left with stirring at room temperature until a clear solution was observed. The solution of $\left[\mathrm{Sm}(\mathrm{hfaa})_{3^{-}}\right.$ (Py-Im)] in THF (5 mL) was added and the mixture was left with stirring overnight. The resulting solution was cast on glass Petri dishes and left for complete evaporation at $50{ }^{\circ} \mathrm{C}$, which resulted in a transparent and smooth thin film.

\subsection{Spectroscopic measurements}

All spectroscopic measurements were performed at RT. The electronic spectra of free Py-Im and the complex were obtained on a Varian Cary 50 spectrophotometer in the 200-600 $\mathrm{nm}$ range. Excitation, emission spectra and decay curves of [Sm(hfaa $\left.)_{3}(\mathrm{Py}-\mathrm{Im})\right]$ complex and their respective hybrid thin films $\left[\mathrm{Sm}(\mathrm{hfaa})_{3^{-}}\right.$ (Py-Im)]@PU_x\% were recorded on an Edinburgh FS5 fluorimeter. All decay curves were fitted to mono-exponential functions using the software supplied with the instrument.

\section{Results and discussion}

The new mixed-ligand complex $\left[\mathrm{Sm}(\mathrm{hfaa})_{3}(\mathrm{Py}-\mathrm{Im})\right]$ was synthesized using a one-pot method (Scheme 1) reported previously for the synthesis of analogous lanthanide complexes. ${ }^{1 a}$ The complex was characterized by elemental analysis, FT-IR spectroscopy and mass spectrometry. The complex is stable to air and moisture and is readily soluble in common organic solvents (except hexane and carbon tetrachloride). The ESI-MS spectrum of the complex in positive mode, in acetone, showed a molecular peak for the species $\left[\mathrm{M}+\mathrm{K}-\left(\mathrm{CH}_{3}\right)_{2} \mathrm{CO}\right]$ with $\mathrm{m} / \mathrm{z}$ value of 948.1 (Fig. S1, ESI $\dagger$ ), confirming the presence of three hfaa and one Py-Im ligand consistent with the formulation of the complex shown in Scheme 1. The FTIR spectrum of the complex (Fig. S2, ESI $\dagger$ ) displays strong absorption peak at $1672 \mathrm{~cm}^{-1}$ due to the $\mathrm{C}=\mathrm{O}$ stretching vibration. The $\nu(\mathrm{C}=\mathrm{N}+\mathrm{C}=\mathrm{C})$ and $\delta(\mathrm{N}-\mathrm{H})$ vibrations of free Py-Im at 1592 and $1440 \mathrm{~cm}^{-1}$ are shifted to higher wavenumbers in the complex, demonstrating ligand coordination to the Sm(III) ion through imidazolic and pyridyl nitrogen atoms. ${ }^{1 a}$ Moreover, a broad absorption band (3300-2350 $\mathrm{cm}^{-1}$ ) with a peak at $3055 \mathrm{~cm}^{-1}$ due to a $\mathrm{N}-\mathrm{H}$ stretching mode in the free Py-Im (Fig. S2, ESI $\dagger$ ) appears as a relatively less strong sharp peak which could be beneficial in suppressing the photoluminescence (PL) quenching.

\subsection{Crystal structure of $\left[\mathrm{Sm}(\mathrm{hfaa})_{3}(\mathrm{Py}-\mathrm{Im})\right]$}

The complex crystallizes in the monoclinic space group $P 2_{1} / n$ with one independent $\left[\mathrm{Sm}(\mathrm{hfaa})_{3}(\mathrm{Py}-\mathrm{Im})\right]$ in the asymmetric unit (Fig. 1a). In the solid-state there is weak intermolecular hydrogen bonding that results in the dimerization of the structure through $\mathrm{N}-\mathrm{H} \cdots \mathrm{O}(\mathrm{H}(3) \cdots \mathrm{O}(1) 2.12 \AA, \mathrm{N}(3) \cdots \mathrm{O}(1)$ $\left.2.920(6) \AA, \mathrm{N}(3)-\mathrm{H}(3) \cdots \mathrm{O}(1) 153.8^{\circ}\right)$ and $\mathrm{N}-\mathrm{H} \cdots \mathrm{F}(\mathrm{H}(3) \cdots \mathrm{F}(3)$ $2.58 \AA, \mathrm{N}(3) \cdots \mathrm{F}(3) 3.108(7) \AA, \mathrm{N}(3)-\mathrm{H}(3) \cdots \mathrm{F}(3) 120.5^{\circ}$ ) hydrogen bonds as illustrated in Fig. 1b. The adjacent $\mathrm{Sm}$ (III) centers are separated by a distance of $8.160(1) \AA$ A. Each Sm(III) centers is in an 8-coordinate $\mathrm{N}_{2} \mathrm{O}_{6}$ environment with two nitrogen atoms from a Py-Im ligand and six oxygen atoms from three hfaa ligands. The coordination geometry of $\mathrm{SmN}_{2} \mathrm{O}_{6}$ is best described as a trigonal dodecahedron. The complex is isomorphous and isostructural with its recently published europium(III) analogue and displays a similar hydrogen bonding pattern. ${ }^{1 a}$

The metal-ligand distances are unremarkable. The Sm-O bond lengths vary from 2.349(3) to 2.454(3) A with an average Sm-O bond length of $2.397 \AA$ comparable to the reported Sm(III) complexes. ${ }^{21}$ The Sm-N1 and Sm-N2 bond lengths are 2.616(4) $\AA$ and 2.501(4) Å, respectively (Table S2, ESI $\dagger$ ). This difference in the Sm-N bond lengths of $0.115 \AA$ is similar to the difference in Eu-N bond lengths of $0.11 \AA$ observed in the Eu analogue, ${ }^{1 a}$ and may be related to the bonding requirements of the Py-Im ligand that coordinates through the nitrogen atoms of linked 5- and 6-membered aromatic rings.

Beyond the dimeric assembly, intermolecular F. F F interactions between the molecules occur (Fig. 1c). The detailed parameters of hydrogen bonds are given in (Table S3, ESI $\dagger$ ). Furthermore, each molecule is linked by weak $\pi-\pi$ stacking interactions $\left[\mathrm{Cg} 1 \cdots \mathrm{Cg} 2^{\mathrm{i}}=3.7460\right.$ (2) $\AA$ and $\mathrm{Cg} 2 \cdots \mathrm{Cg} 3^{\mathrm{i}}=3.7095$ (2) $\AA$; $\mathrm{Cg} 1, \mathrm{Cg} 2$ and $\mathrm{Cg} 3$ are the centroids of the N2/C6/N3/C7/ $\mathrm{C} 12, \mathrm{~N} 1 / \mathrm{C} 1-\mathrm{C} 5$ and $\mathrm{C} 7-\mathrm{C} 12$ rings (Fig. S3, ESI $\dagger$ ), respectively. The symmetry code is $\mathrm{i}=-x+1,-y+1,-z+1]$, forming a $3-\mathrm{D}$ structure.

The three-dimensional Hirshfeld surface (HS) map generated for the complex is presented in Fig. 2a. Hirshfeld surface analysis is important in assessing the interactions in the crystal packing. In the maps in Fig. $2 \mathrm{a}$, red areas are related to $\mathrm{N}-\mathrm{H} \cdots \mathrm{O}$ intermolecular contacts. In the Hirshfeld surface maps, the contacts shown in red highlight the intermolecular interactions with distances shorter than the sum of the van der Waals radii. ${ }^{18} 2 \mathrm{D}$ fingerprint plots (FPs) have been obtained from the Hirshfeld surface (HS) by plotting the fraction of points on the surface as a function of $\left(d_{\mathrm{e}}, d_{i}\right)$. Herein, $d_{\mathrm{e}}$ and $d_{i}$ represent the distances from a point on the HS to the nearest atoms outside and inside the surface, respectively. F $\cdots \mathrm{H} / \mathrm{H} \cdots \mathrm{F}, \mathrm{F} \cdots \mathrm{F}, \mathrm{F} \cdots \mathrm{C} /$ $\mathrm{C} \cdots \mathrm{F}, \mathrm{C} \cdots \mathrm{H} / \mathrm{H} \cdots \mathrm{C}$ and $\mathrm{H} \cdots \mathrm{H}$ interactions exist in the FPs of complex (Fig. 2b). The $\mathrm{F} \cdots \mathrm{H} / \mathrm{H} \cdots \mathrm{F}$ contacts represent the largest relative contribution, amounting to $39.4 \%$ in the complex (appearing as two narrow long spikes). The $\mathrm{C} \cdot \mathrm{H} / \mathrm{H} \cdots \mathrm{C}$ contacts $(7.4 \%)$ appear as two broad short spikes. Because of the replacement of 
(a)

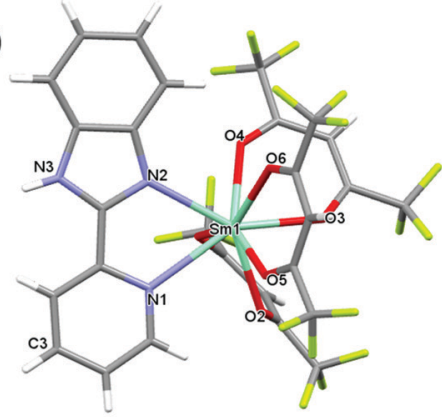

(b)

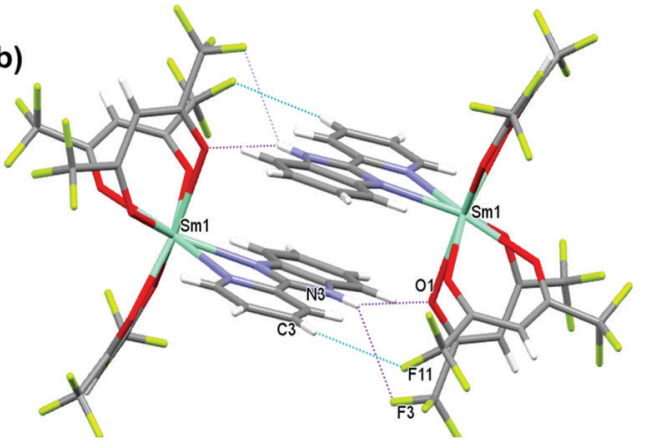

(c)

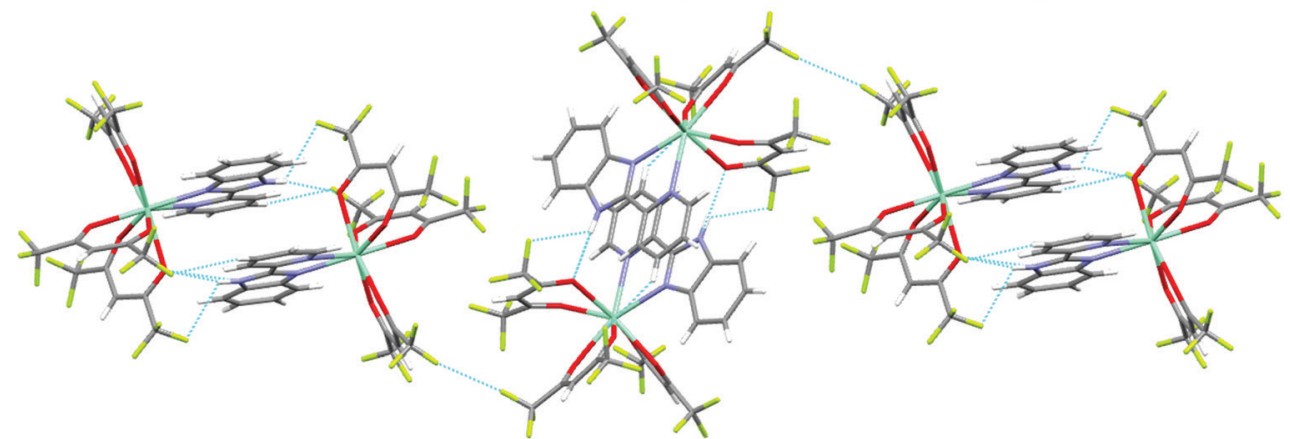

Fig. 1 (a) A view of the asymmetric unit of $\left[\mathrm{Sm}(\mathrm{hfaa})_{3}(\mathrm{Py}-\mathrm{Im})\right]_{2}$ and (b) comparative view of $\left[\mathrm{Sm}(\mathrm{hfaa})_{3}(\mathrm{Py}-\mathrm{Im})\right]$ represented via $\mathrm{N}-\mathrm{H} \ldots \mathrm{O}$ and $\mathrm{N}-\mathrm{H} \ldots \mathrm{F}$ intermolecular interactions. Dotted pink and blue lines depict the intermolecular interactions (c) packing diagram of the crystal exhibiting $F \ldots F$ interaction between hfaa ligands.

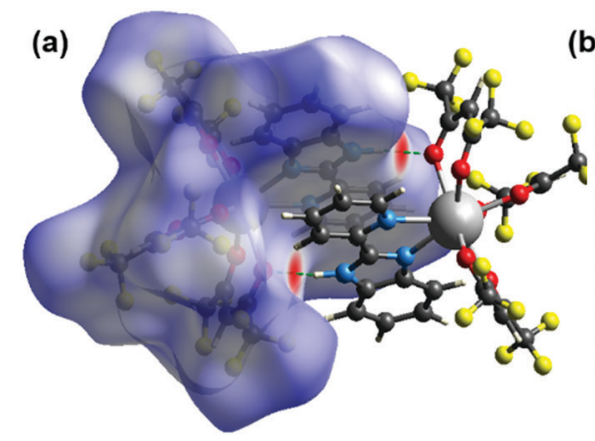

(b)

(c)
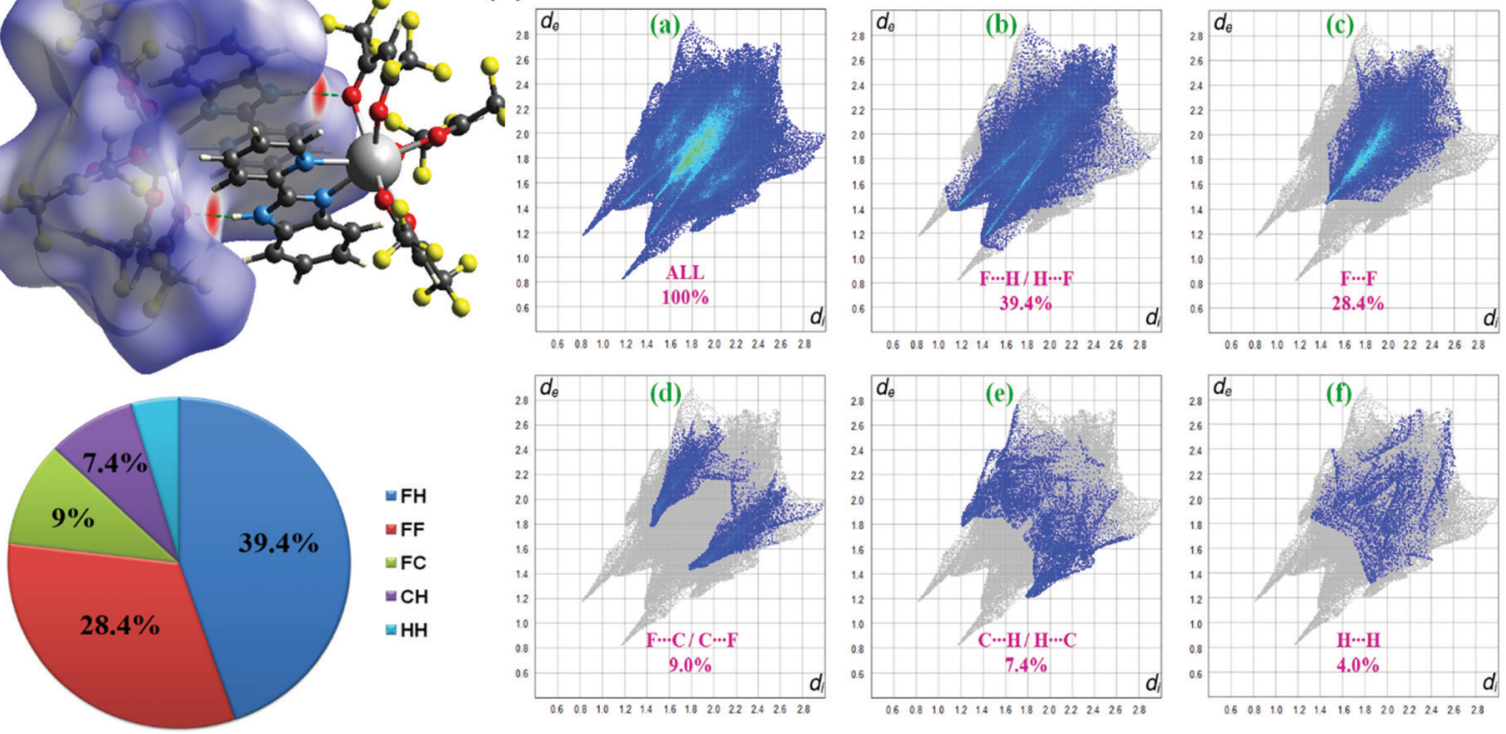

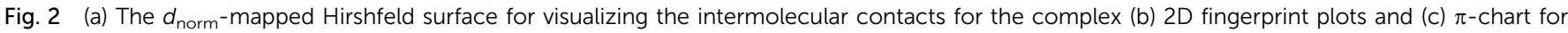
complex showing the percentage contribution of interactions.

$\mathrm{CH}_{3}$ groups by $\mathrm{CF}_{3}$ groups in the ligands, compared to other studies in the literature, ${ }^{2 c, 22}$ in this complex, the contribution of $\mathrm{H} \cdots \mathrm{H}$ interactions (4\%) to the total HS is quite low.

\subsection{Thermal analysis}

An important factor for the use of lanthanide-based hybrid materials in optoelectronic devices is their high thermal stability.
The thermal stability of the Sm(III) complex was determined simultaneously by thermogravimetric analysis (TGA) and differential thermal analysis (DTA) as shown in Fig. 3. The thermogram exhibits a one-step weight loss due to the removal of the coordinated organic ligands (hfaa and Py-Im) with the decomposition temperature $\left(T_{\mathrm{d}}\right) \approx 224{ }^{\circ} \mathrm{C}$. The DTA curve shows one sharp endothermic peak at $195{ }^{\circ} \mathrm{C}$ representing the melting point $\left(T_{\mathrm{m}}\right)$ of the complex. 


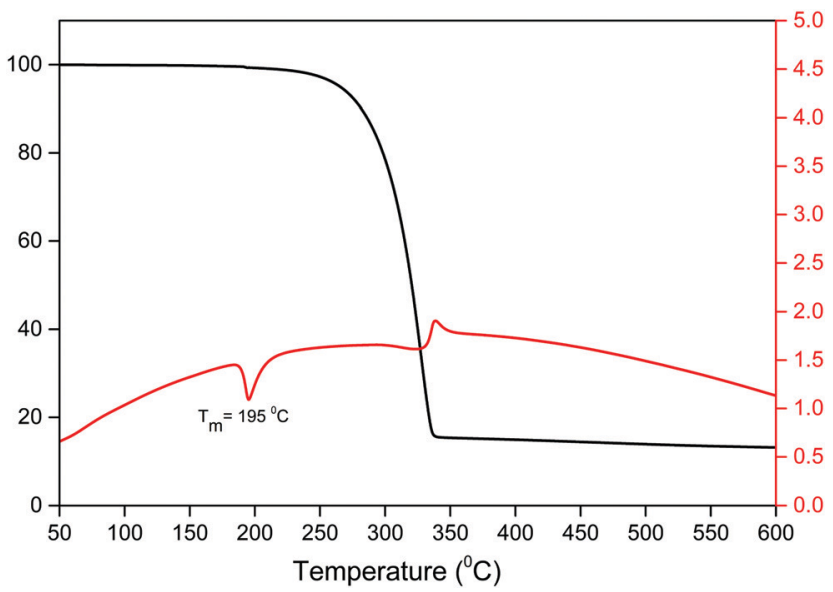

Fig. 3 TGA/DTA curve of the complex under $\mathrm{N}_{2}$ atmosphere.

The high $T_{\mathrm{d}}$ and $T_{\mathrm{m}}$ of the complex could be attributed to the hydrogen bonding and $\pi-\pi$ stacking interactions in the complex in the solid-state. Moreover, the thermogram does not show any weight loss in the region $50-180{ }^{\circ} \mathrm{C}$ implying that it has no coordinated water/solvent molecules confirming the results of IR spectroscopic study.

\subsection{Photophysical properties}

For strongly luminescent lanthanide complexes, the coordinated ligand(s) need to have some necessary prerequisites, namely; a strong light absorption properties that would result in strong light absorbing complexes and appropriate energy differences $(\Delta E)^{23}$ between the ${ }^{3} \pi-\pi^{*}$ states of the coordinated organic ligand(s) and the emitting levels of Ln(III) ions. In order to check the first requirement, the electronic spectra of the free Py-Im ligand and the complex were recorded in DCM (Fig. S4, ESI $\dagger$ ). The spectrum of the free Py-Im displays an intense absorption at $310 \mathrm{~nm} .{ }^{1 a}$ The spectrum of the complex exhibits overlapping absorption bands of the Py-Im and hfaa with maxima at $298 \mathrm{~nm}$ with molar absorption coefficient $6.49 \times 10^{4} \mathrm{M}^{-1} \mathrm{~cm}^{-1}$ suggesting that the complex has strong light absorbing capability and hence potential for efficient PL. For the second parameter in the present complex, $\Delta E$ was calculated from the ${ }^{3} \pi-\pi^{*}$ transition of the organic ligand(s) coordinated $\left({ }^{3} \pi-\pi^{*} \approx 21930 \mathrm{~cm}^{-1}\right.$ for $\mathrm{hfaa}^{24}$ and $20576 \mathrm{~cm}^{-1}$ for Py-Im, ${ }^{1 a}$ respectively) to the central Sm(III) ion. The trivalent samarium ion has three potential excited states ${ }^{4} \mathrm{G}_{5 / 2}\left(\approx 17924 \mathrm{~cm}^{-1}\right),{ }^{4} \mathrm{~F}_{3 / 2}\left(\approx 18332 \mathrm{~cm}^{-1}\right)$ and ${ }^{4} \mathrm{G}_{7 / 2}\left(\approx 20014 \mathrm{~cm}^{-1}\right)$, respectively that can receive energy from ${ }^{3} \pi-\pi^{*}$ states of the organic ligands. ${ }^{25}$ It is generally believed that after receiving energy from the ${ }^{3} \pi-\pi^{*}$ states, the energy is transferred to ${ }^{4} \mathrm{G}_{7 / 2}$ and ${ }^{4} \mathrm{~F}_{3 / 2}$ which finally relax the absorbed energy to ${ }^{4} \mathrm{G}_{5 / 2}$ state, which in turn, undergoes radiative processes leading to emission from ${ }^{4} \mathrm{G}_{5 / 2}$ state. ${ }^{26}$ The values of $\Delta E\left({ }^{3} \pi-\right.$ $\left.\pi^{*}-{ }^{4} \mathrm{G}_{5 / 2}\right), \Delta E\left({ }^{3} \pi-\pi^{*}-{ }^{4} \mathrm{~F}_{3 / 2}\right)$ and $\Delta E\left({ }^{3} \pi-\pi^{*-}{ }^{4} \mathrm{G}_{7 / 2}\right)$ are 4006,3598 and $1916 \mathrm{~cm}^{-1}$, respectively for hfaa while 2652,2244 and 562 $\mathrm{cm}^{-1}$, respectively, for Py-Im. These energy differences suggest that the present complex should display strong emission at RT. In view of this we first analyzed the steady-state excitation and emission spectra as shown in Fig. 4. The excitation spectrum of the complex was obtained by monitoring the most intense emission transition appearing at $648 \mathrm{~nm}$ in the emission spectrum of the complex. The excitation spectrum in the 300-550 nm exhibits broadband and some intra-configurational transitions as shown in Fig. 4 a at $406 \mathrm{~nm}, 418 \mathrm{~nm}, 477 \mathrm{~nm}$ and $527 \mathrm{~nm}$, and are assigned ${ }^{27}$ to ${ }^{4} \mathrm{M}_{21 / 2},{ }^{4} \mathrm{~K}_{11 / 2},{ }^{6} \mathrm{P}_{3 / 2},{ }^{4} \mathrm{~F}_{7 / 2},{ }^{4} \mathrm{~L}_{13 / 2} \leftarrow{ }^{6} \mathrm{H}_{5 / 2},{ }^{4} \mathrm{M}_{19 / 2}$, $\left({ }^{6} \mathrm{P},{ }^{4} \mathrm{P}\right)_{5 / 2} \leftarrow{ }^{6} \mathrm{H}_{5 / 2},{ }^{4} \mathrm{I}_{11 / 2},{ }^{4} \mathrm{M}_{19 / 2},{ }^{4} \mathrm{I}_{9 / 2} \leftarrow{ }^{6} \mathrm{H}_{5 / 2}$ and ${ }^{4} \mathrm{~F}_{3 / 2} \leftarrow{ }^{6} \mathrm{H}_{5 / 2}$, respectively.

The emission spectrum of the complex at RT was obtained by choosing an excitation wavelength from the excitation spectrum to maximize the emission. The spectrum displays four wellresolved emission transitions at $564 \mathrm{~nm}, 605 \mathrm{~nm}, 648 \mathrm{~nm}$ and $714 \mathrm{~nm}$, which are assigned to ${ }^{4} \mathrm{G}_{5 / 2} \rightarrow{ }^{6} \mathrm{H}_{5 / 2}$ (forbidden transition), ${ }^{4} \mathrm{G}_{5 / 2} \rightarrow{ }^{6} \mathrm{H}_{7 / 2}$ (magnetic dipole (MD) transition), ${ }^{4} \mathrm{G}_{5 / 2} \rightarrow{ }^{6} \mathrm{H}_{9 / 2}$ (electric dipole (ED) transition) and ${ }^{4} \mathrm{G}_{5 / 2} \rightarrow$ ${ }^{6} \mathrm{H}_{11 / 2}$ (forbidden transition), respectively (Fig. $4 \mathrm{~b}$ ). Out of the four emission transitions in the spectrum, ED ${ }^{4} \mathrm{G}_{5 / 2} \rightarrow{ }^{6} \mathrm{H}_{9 / 2}$
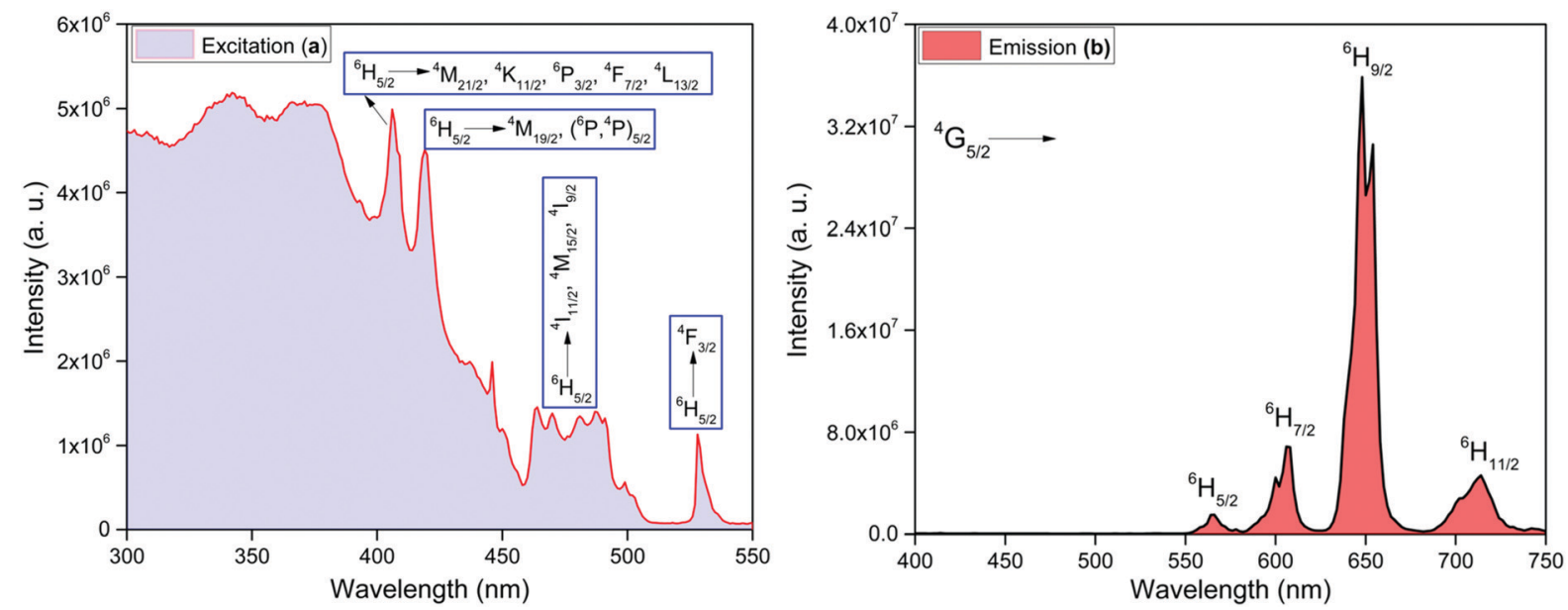

Fig. 4 Solid-state (a) excitation spectrum monitored at $\lambda_{\mathrm{em}} \approx 648 \mathrm{~nm}$ and (b) corrected emission spectrum of the complex monitored at $\lambda_{\mathrm{ex}} \approx 374 \mathrm{~nm}$ at RT. 
Table 1 Photophysical parameters of the complex in different media at RT

\begin{tabular}{lllllll}
\hline Medium & FWHM $^{a}(\mathrm{~nm})$ & $R_{\mathrm{Sm}}{ }^{b}$ & $\tau_{\text {exp. }}(\mu \mathrm{s})$ & CIE $(x ; y)$ & $Q_{\mathrm{Sm}}{ }^{c}(\%)$ \\
\hline Solid-state & 11.83 & 6.00 & $204.47 \pm 4.043$ & $0.6532 ; 0.3336$ & Color \\
DCM & 12.25 & 2.75 & $105.50 \pm 0.143$ & $0.6071 ; 0.3568$ & Brilliant red & Orange \\
ACN & 11.13 & 2.70 & $42.75 \pm 0.055$ & $0.5607 ; 0.3167$ & Dark-coral & 3.40 \\
Acetone & 7.61 & 2.80 & $37.80 \pm 0.187$ & $0.4347 ; 0.2328$ & Rose-pink & 1.37 \\
THF & 6.55 & 2.47 & $35.01 \pm 0.187$ & $0.3451 ; 0.2355$ & Light-violet & 1.22 \\
EtOH & 7.96 & 2.20 & $20.39 \pm 0.041$ & $0.4409 ; 0.2623$ & Pale pink \\
MeOH & 8.02 & 2.39 & $14.27 \pm 0.033$ & $0.3634 ; 0.2144$ & Violet
\end{tabular}

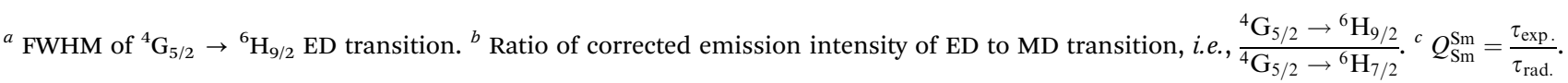

hypersensitive transition dominates with the full width at half maxima $(\mathrm{FWHM}) \approx 11.83 \mathrm{~nm}$ (Table 1) followed by the MD transition. It is well-established that the ratio $\left(R_{\mathrm{Ln}}\right)$ of the corrected ED to MD emission transition gives clues regarding the coordination symmetry and polarizability of the local environment around the $\operatorname{Ln}(\mathrm{III})$ ion in a given complex i.e., $R_{\mathrm{Sm}}=\mathrm{ED}\left({ }^{4} \mathrm{G}_{5 / 2} \rightarrow{ }^{6} \mathrm{H}_{9 / 2}\right) / \mathrm{MD}\left({ }^{4} \mathrm{G}_{5 / 2} \rightarrow{ }^{6} \mathrm{H}_{7 / 2}\right)$. The higher intensity of the ED transition and high $R_{\mathrm{Sm}}$ value of 6.00 for the present complex suggests a low molecular symmetry of the coordination sphere and that $\mathrm{Sm}$ (III) ion in the complex sits in a highly polarizable environment. The $R_{\mathrm{Sm}}$ value is comparable to values reported for similar $\mathrm{Sm}(\mathrm{III})$ complexes. $^{2 c, 8 a}$ It worth noting that there is no residual ligand fluorescence in the 400-500 $\mathrm{nm}$ range suggesting that the energy absorbed by the organic ligands is efficiently transferred to Sm(III) emitting state as can be seen in Fig. $4 \mathrm{~b}$ and colorful emission spectrum in Fig. S5, ESI, $\dagger$ as expected by the energy gap law. To get information about the emitted color from the solid complex, the CIE color coordinates $(x ; y)$ were calculated from the steady-state emission spectrum and the results are summarized in Table 1 . As can be seen from the magnified CIE color diagram in Fig. 5, the complex emits intense red emission with color coordinates $x=0.6532$; $y=0.3336$, which are similar to National Television System Committee (NTSC) $(x=0.67 ; y=0.33)$. It is important to emphasize that there are only few reported $\left[\mathrm{Sm}(\beta \text {-diket. })_{3}\right.$ (ancillary ligand)] complexes that display an intense red emission. ${ }^{6,8 a, b}$

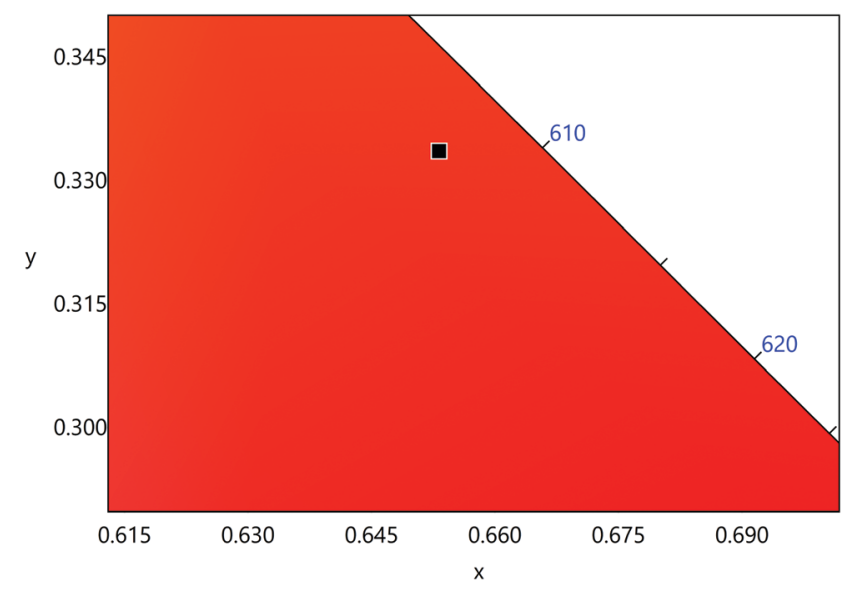

Fig. 5 A magnified view of the CIE color diagram showing the intense red emission from the complex in the solid-state at RT.
In addition to the steady-state emission, we also carried out the time-resolved PL decay dynamics. The PL lifetime $\left(\tau_{\exp }\right)$ of the complex in the solid-state was measured at RT (Fig. S6 (ESI $\dagger$ ) and Table 1) by monitoring the emission decay curve within ${ }^{4} \mathrm{G}_{5 / 2} \rightarrow{ }^{6} \mathrm{H}_{9 / 2}$ transition at $648 \mathrm{~nm}$. The decay curve fits very well with mono-exponential behavior, indicating the presence of only emitting $\mathrm{Sm}$ (III) center in the solid-state. The complex displays remarkably long $\tau_{\text {exp. }} \approx 204.47 \pm 4.043 \mu$ s, which could be attributed to optimum $\Delta E$ between $\mathrm{Sm}$ (III) emitting state and prime hfaa and ancillary Py-Im ligands. Furthermore, the minimization of high energy oscillator strength of the $\mathrm{N}-\mathrm{H}$ by intermolecular interactions i.e., $\mathrm{N}-\mathrm{H} \cdots \mathrm{O}$ and $\mathrm{N}-\mathrm{H} \cdots \mathrm{F}$ as shown in Fig. $1 \mathrm{~b}$ and was complimented by the FTIR results, which show a relatively less strong sharp peak for $\mathrm{N}-\mathrm{H}$ oscillator (Fig. S2, ESI $\dagger$ ). Moreover, as determined by $\mathrm{HS}$ maps, the $\mathrm{F} \cdots \mathrm{H} / \mathrm{H} \cdots \mathrm{F}$ largest relative contribution, amounting to $39.4 \%$ in the present complex further diminishes the role of another high energy oscillator enhancing the PL lifetime. Thus, HS analysis and 2-D fingerprint were very useful for understanding the contribution of intermolecular interactions in determining the PL properties. The PL lifetime of the present complex is higher than most of the known highly efficient $\left[\mathrm{Sm}(\beta \text {-diket. })_{3}\right.$ (ancillary ligand)] complexes such as $\left[\mathrm{Sm}(\mathrm{hfaa})_{3}(\mathrm{TPPO})_{2}\right] \approx 97 \mu \mathrm{s},{ }^{8 a}\left[\mathrm{Sm}(\mathrm{hfaa})_{3}(\mathrm{phen})_{2}\right] \approx 37 \mu \mathrm{s}^{8 a}$ and $54-56 \mu \mathrm{s},{ }^{6}\left[\mathrm{Sm}_{2}(\mathrm{BTP})_{3}(\text { phen })_{2}\right] \approx 100 \mu \mathrm{s},{ }^{28}\left[\mathrm{Sm}(\mathrm{hfaa})_{3^{-}}\right.$ $(t \mathrm{Bu}$-xantpo $)] \approx 150 \mu \mathrm{s}^{8 c}$ and is lower only two longest and efficient complexes $\left[\mathrm{Sm}(\mathrm{hfaa})_{3}(\right.$ pybox $\left.)\right] \approx 390 \mu \mathrm{s}^{8 b}$ and $\left[\mathrm{Sm}(\mathrm{hfaa})_{3^{-}}\right.$ (dpepo) $] \approx 280 \mu \mathrm{s}^{8 c}$ until now, where TPPO = triphenylphosphine oxide; phen = 1,10-phenanthroline; BTP = 1,3-bis(4,4,4-trifluoro1,3-dioxobutyl)phenyl; pybox = bis(oxazolinyl)pyridine; dpepo = bis[(2-diphenylphosphoryl)phenyl $]$ ether; $t$ Bu-xantpo $=4,5$-bis(di-tert-butylphosphoryl)-9,9-dimethylxanthene. The intrinsic quantum yield $\left(Q_{\mathrm{Sm}}^{\mathrm{Sm}}\right)$ of the complex is calculated by the following general expression 2 and the obtained data is summarized in Table 1:

$$
Q_{\mathrm{sm}}^{\mathrm{sm}}=\frac{\tau_{\mathrm{exp}}}{\tau_{\mathrm{rad}}}
$$

where $\tau_{\text {rad }}$ is the calculated natural radiative lifetime of Sm(III) ion $(3100 \mu \mathrm{s})$ reported in the literature. ${ }^{29}$

\subsection{Effect of medium on PL properties and color.}

$\Delta E$ between the emitting level and the next lower energy level in $\mathrm{Sm}(\mathrm{III})$ is $\approx 7400 \mathrm{~cm}^{-1} \cdot{ }^{30}$ Therefore PL quenching is inevitable by the high energy oscillators $\left(\mathrm{O}-\mathrm{H} \sim 3300-3500 \mathrm{~cm}^{-1}\right.$, 
$\mathrm{N}-\mathrm{H} \sim 3100-3300 \mathrm{~cm}^{-1}$ and $\left.\mathrm{C}-\mathrm{H} \sim 2950 \mathrm{~cm}^{-1}\right)^{31}$ of the solvents as well as oscillators within the coordinated ligands. In order to see the effect of the different solvents on the PL properties of the complex, we first analyzed the excitation spectra in different solvents. The excitation spectra in different solvents were obtained as discussed above and are shown in Fig. S7, ESI. $\dagger$ The spectra displayed broadband between 310$380 \mathrm{~nm}$ with the obvious change in intensity and follows the trend: DCM $>$ ACN $>$ Acetone $>$ THF $>$ EtOH $>\mathrm{MeOH}$.

Steady-state emission spectra as shown in Fig. 6 were obtained by exciting them to their $\lambda_{\mathrm{Ex}}^{\max }$ and exhibit typical emission transitions of Sm(III) ion as observed and discussed in the case of solid-state. The emission transitions follow the similar trend as observed ${ }^{4} \mathrm{G}_{5 / 2} \rightarrow{ }^{6} \mathrm{H}_{9 / 2} \gg{ }^{4} \mathrm{G}_{5 / 2} \rightarrow{ }^{6} \mathrm{H}_{7 / 2}>$ ${ }^{4} \mathrm{G}_{5 / 2} \rightarrow{ }^{6} \mathrm{H}_{5 / 2}>{ }^{4} \mathrm{G}_{5 / 2} \rightarrow{ }^{6} \mathrm{H}_{11 / 2}$. Moreover, a concomitant residual ligand fluorescence is also observed (Fig. 6 and for a

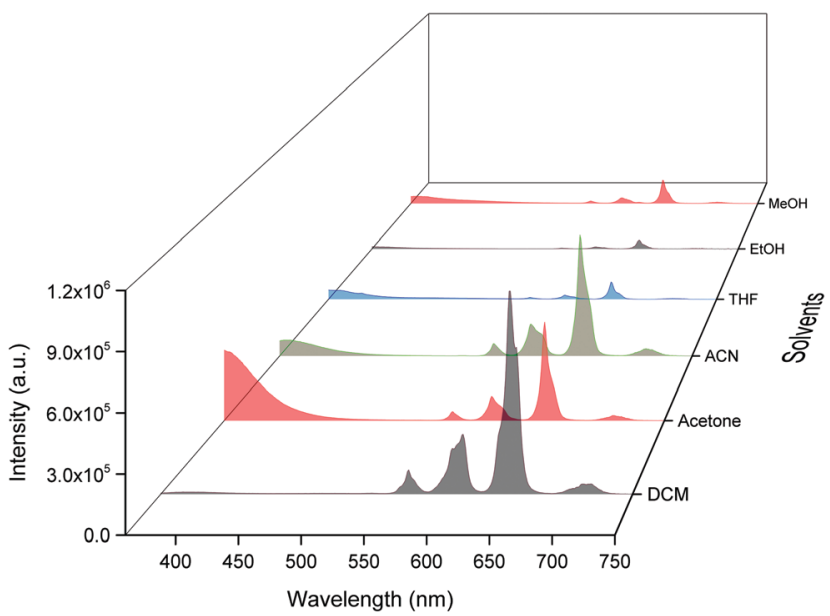

Fig. 6 Corrected emission spectra of the complex in different solvents at RT.

(a)

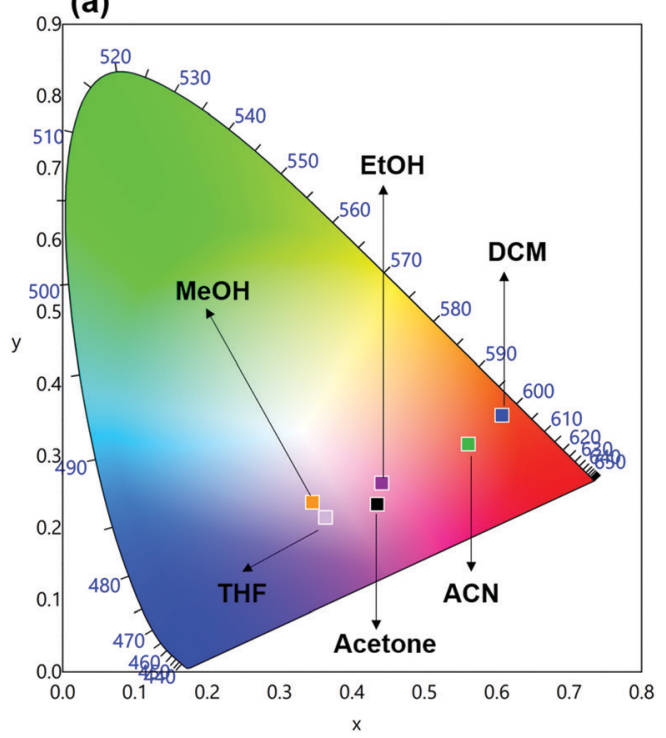

clearer understanding each emission spectrum is separately shown in Fig. S8-S13, ESI $\dagger$ ), with their observed color maxima which is absent in the solid-state and increased by changing the solvents. This could be attributed to the interaction of high energy oscillators of solvent(s) and quenching of the ${ }^{3} \pi \pi^{*}$ energy by the dipole-dipole coupling of the ligands (hfaa and Py-Im) with the solvent ${ }^{32}$ and thus effecting the overall ET from the ligand(s) to Sm(III) ion. It is well known that the intensity of hypersensitive transition of $\operatorname{Ln}$ (III) ions is highly sensitive to the surrounding chemical environment and changes with the change in the solvent. The intensity of the hypersensitive ${ }^{4} \mathrm{G}_{5 / 2} \rightarrow{ }^{6} \mathrm{H}_{9 / 2}$ transition in different solvents follows the order: DCM $\gg$ ACN $>$ Acetone $>$ THF $>\mathrm{MeOH}>$ EtOH. The change in the intensity of the $\mathrm{ED}{ }^{4} \mathrm{G}_{5 / 2} \rightarrow{ }^{6} \mathrm{H}_{9 / 2}$ transition is further reflected in the $R_{\mathrm{Sm}}$ values 2.75-2.20 on going from DCM to $\mathrm{MeOH}$ (Table 1 ). In order to understand more clearly the effect of different solvents, we determined $\tau_{\text {exp. }}$ in each solvent. In each case decay profiles (Fig. S14-S19, ESI $\dagger$ ) satisfactory fit to mono-exponential and data obtained are gathered in Table 1 . As expected, $\tau_{\exp }$ in solution $(105.50 \pm 0.143$ to $14.27 \pm 0.033 \mu \mathrm{s})$ is shorter than in the solidstate $(204.47 \pm 4.043 \mu \mathrm{s})$. The relatively shorter $\tau_{\text {exp. value in DCM }}$ with almost $50 \%$ decrease could be due to: (i) a smaller energy gap of $\Delta E=7400 \mathrm{~cm}^{-1}$ between the ${ }^{4} \mathrm{G}_{5 / 2}$ and the next lower energy level that matches to the 1 st and 2 nd overtone of $\mathrm{C}-\mathrm{H}$ oscillator $\left(\nu_{\mathrm{C}-\mathrm{H}} \approx 2950 \mathrm{~cm}^{-1}\right)$ (ii) quenching of the ${ }^{3} \pi \pi^{*}$ energy by the dipole-dipole coupling of the ligands (hfaa and Py-Im) with the solvent molecule(s) that decreases the overall ET efficiency and thus leads to RFL in the range between 350-500 nm (Fig. S8-S13, ESI $\dagger$ ) and (iii) conversion of the $\mathrm{M}_{2} \mathrm{~L}_{2}$ assembly into ML structure that diffuses randomly in the solution with free $\mathrm{N}-\mathrm{H}$ oscillator which results in more efficient quenching in polar solvents. The $\tau_{\text {exp. }}$ further dropped to $20.39 \pm 0.041$ and $14.27 \pm$ $0.033 \mu$ s on changing the solvent to $\mathrm{EtOH}$ and $\mathrm{MeOH}$, respectively. This drop in $\tau_{\text {exp. }}$ is due to presence of $\mathrm{O}-\mathrm{H}$ oscillator $\left(\nu_{\mathrm{O}-\mathrm{H}} \approx\right.$ $3300-3500 \mathrm{~cm}^{-1}$ ) that matches the 1st vibrational overtone and

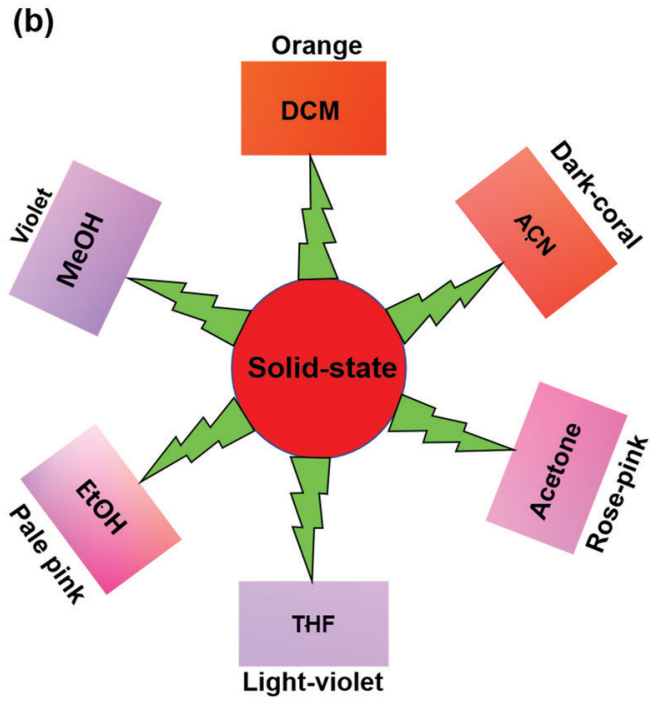

Fig. 7 (a) 1931 CIE Chromaticity diagram and (b) a magnified CIE showing the exact emitted color of the complex in different solvents at RT. 
thus the Franck-Condon factor becomes more favorable. This results in higher quenching compared to DCM and other solvents and less efficient ET, which could be seen (Fig. 5 and separate emission diagram Fig. S8-S13, ESI $\dagger$ ) with concomitant decrease in the intensity of hypersensitive ${ }^{4} \mathrm{G}_{5 / 2} \rightarrow{ }^{6} \mathrm{H}_{9 / 2}\left(I_{\text {Red }}\right)$ in the red

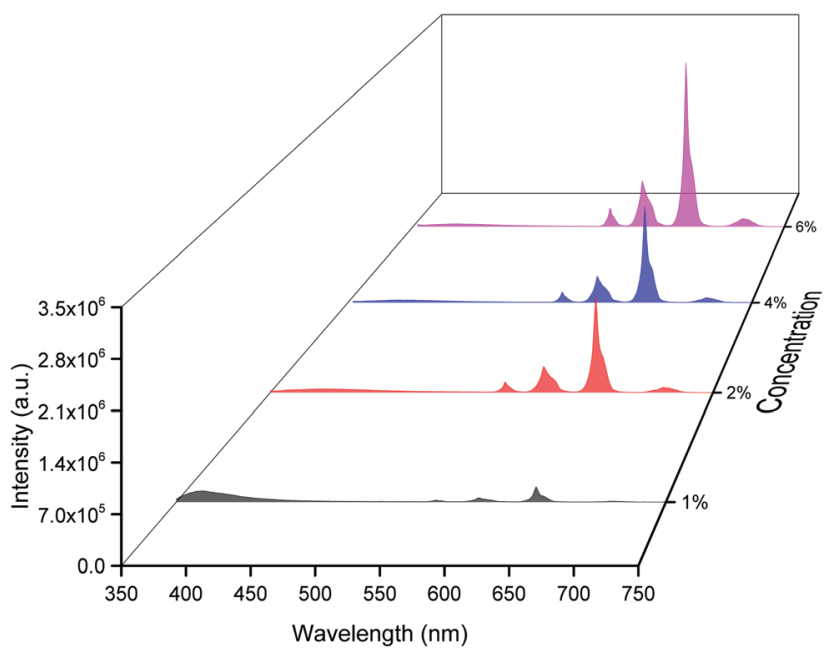

Fig. 8 Corrected emission spectra of [Sm(hfaa) $\left.)_{3}(\mathrm{Py}-\mathrm{Im})\right] @ \mathrm{QPU}$ at different doping concentrations at RT. region and simultaneous increase in intensity of RFL $\left(I_{\mathrm{RFL}}\right)$ in the blue region. The change in the $I_{\text {Red }}$ and $I_{\mathrm{RFL}}$ will subsequently effect the emitted color of the complex. In order to see the emitted color in different solvents, we calculated the CIE color coordinates $(x ; y)$ from the emission spectra and the obtained color coordinates are shown in Table 1 and Fig. 7. It is clear from the CIE color diagram that the present complex displays different colors in different solvents such as orange color in DCM and violet in $\mathrm{MeOH}$ (Fig. 7b) due to increase of $I_{\mathrm{RFL}}$.

The $Q_{\mathrm{Sm}}^{\mathrm{Sm}}$ of the complex in each solvent was calculated (Table 1) and follows the trend: DCM $\gg$ ACN $>$ Acetone $>$ $\mathrm{THF}>\mathrm{EtOH}>\mathrm{MeOH}$, respectively, which is directly related to $\tau_{\text {exp. }}$ Looking at these interesting PL properties, i.e., remarkably long $\tau_{\text {exp. }}$ in the solid-state and color tunability from orange to violet of the present complex in different solvents, we decided to develop hybrid thin films by encapsulating it in a PU polymer matrix. The encapsulation of lanthanide complex into polymer matrix usually increases the thermal and photostability coupled with the enhanced mechanical strength of the developed hybrid material, a necessary prerequisite for optoelectronic applications. $^{2 a, 33}$ The hybrid and flexible thin films were developed by doping different concentrations such as $1 \%, 2 \%, 4 \%$ and $6 \%$ of the complex into the PU polymer matrix assuming that it would give us color-tunable materials. The PL properties of the flexible

Table 2 Photophysical parameters of hybrid thin films of [Sm(hfaa) $\left.{ }_{3}(\mathrm{Py}-\mathrm{Im})\right]$ at RT

\begin{tabular}{lllllll}
\hline Medium (\%) & FWHM $^{a}(\mathrm{~nm})$ & $R_{\mathrm{Sm}}{ }^{b}$ & $\tau_{\exp }(\mu \mathrm{s})$ & CIE $(x ; y)$ & $Q_{\mathrm{Sm}}^{\mathrm{Sm} c}(\%)$ \\
\hline 1 & 5.66 & 1.99 & $43.55 \pm 0.274$ & $0.3380 ; 0.2162$ & Color & Violet \\
2 & 5.90 & 2.45 & $46.85 \pm 0.240$ & $0.5028 ; 0.3080$ & Coral pink & 1.40 \\
4 & 5.72 & 2.40 & $51.81 \pm 0.149$ & $0.5295 ; 0.3203$ & Light coral & 1.51 \\
6 & 5.84 & 2.41 & $54.14 \pm 0.131$ & $0.5666 ; 0.3408$ & Light orange & 1.74
\end{tabular}

${ }^{a}$ FWHM of ${ }^{4} \mathrm{G}_{5 / 2} \rightarrow{ }^{6} \mathrm{H}_{9 / 2}$ ED transition. ${ }^{b}$ Ratio of corrected emission intensity of ED to MD transition, i.e., $\frac{{ }^{4} \mathrm{G}_{5 / 2} \rightarrow{ }^{6} \mathrm{H}_{9 / 2}}{{ }^{4} \mathrm{G}_{5 / 2} \rightarrow{ }^{6} \mathrm{H}_{7 / 2}} Q_{\mathrm{Sm}}^{\mathrm{Sm}}=\frac{\tau_{\mathrm{exp}}}{\tau_{\mathrm{rad}} .}$
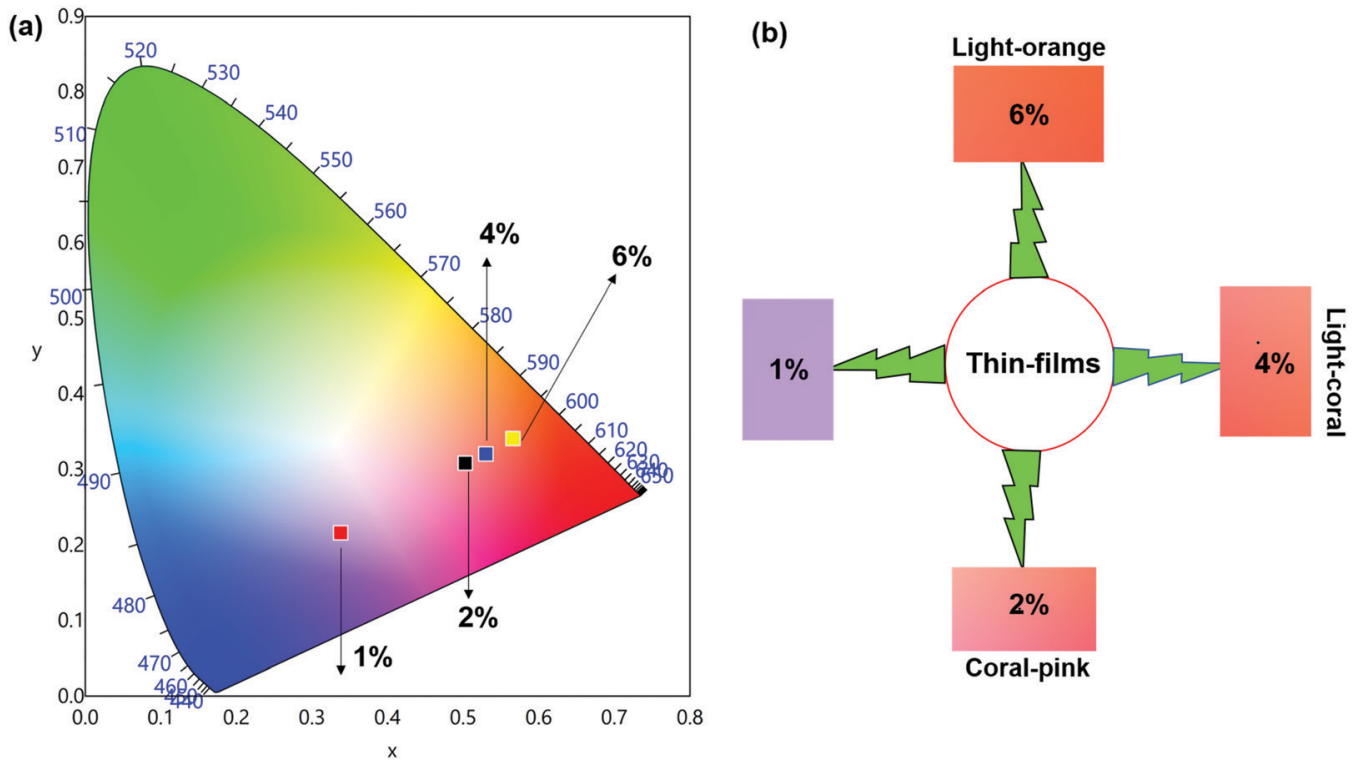

Fig. 9 (a) 1931 CIE Chromaticity diagram and (b) a magnified CIE showing the exact emitted color of [Sm(hfaa) $3($ Py-Im)]@PU_x\% in different doping concentration at RT. 
thin films were analyzed by the steady-state excitation and emission spectra. The excitation spectra of thin films displayed similar broadband as observed in the solid-state and in solution (Fig. S20, ESI $\dagger$ ).

The emission spectra of the hybrid thin films obtained by exciting at $\lambda_{\mathrm{Ex}}^{\max }$ displayed typical metal centered emission transitions (discussed above) as observed in the solid-state and in solution as shown in Fig. 8 and the data obtained is shown in Table 2. Hypersensitive ${ }^{4} \mathrm{G}_{5 / 2} \rightarrow{ }^{6} \mathrm{H}_{9 / 2}$ emission transition dominates the spectra and is narrower in hybrid thin films with
FWHM in 5.66-5.90 nm indicating the higher potential of the developed hybrid thin film for optoelectronic applications. $R_{\mathrm{Sm}}$ values remain in the range of 1.99-2.45 (Table 2) as observed in different solvents suggesting that incorporation of the complex in the PU polymer matrix does not alter the symmetry and coordination sphere of the bare complex, so it does not lose its structural identity. The $\tau_{\text {exp. }}$ of hybrid thin films were determined as discussed above and the obtained data is gathered in Table 2 while the decay curves together with fitted curves are shown in Fig. S21-S24, ESI. $\dagger$ The lifetime of the hybrid thin

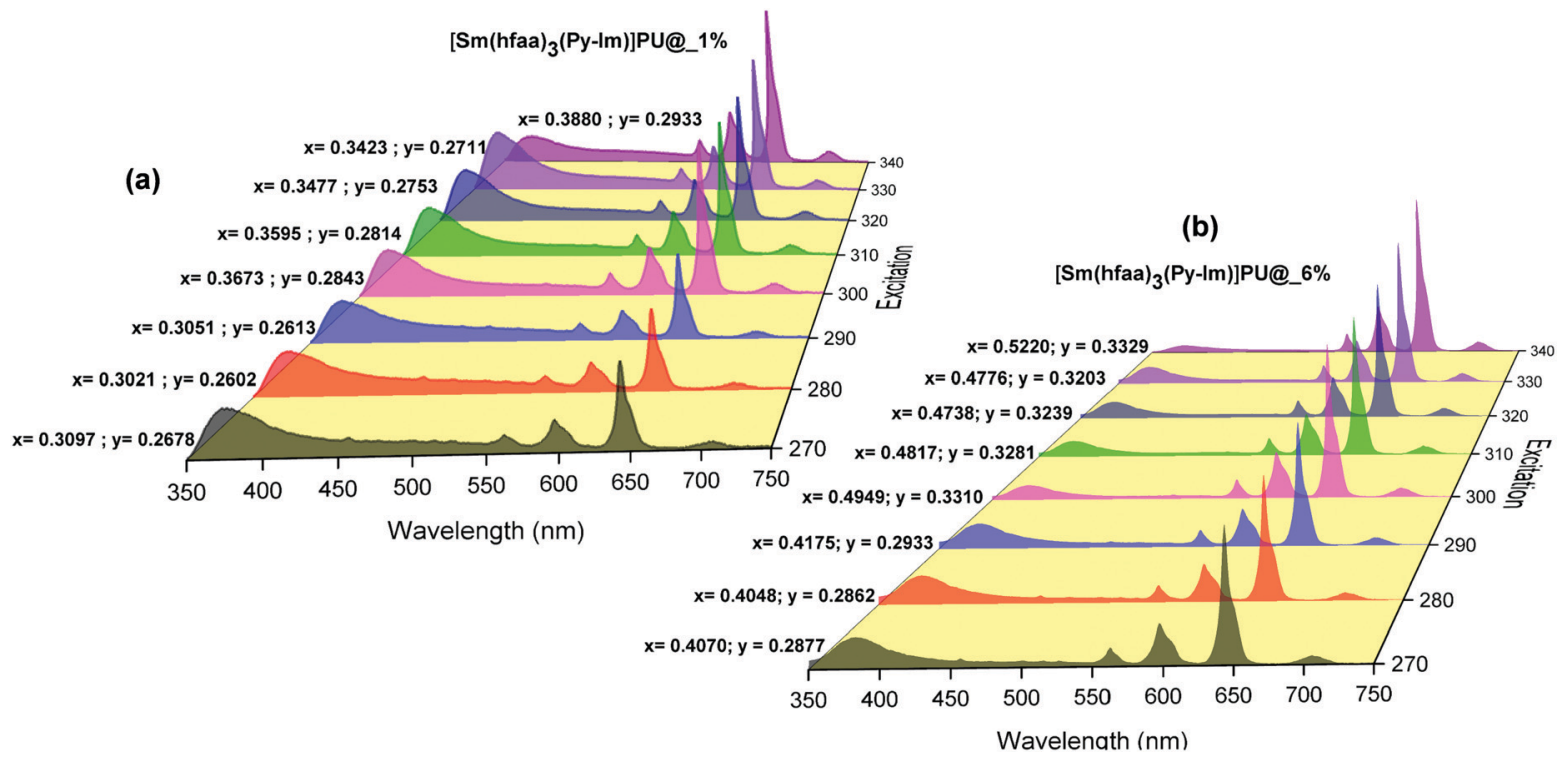

Fig. 10 Corrected emission spectra of hybrid thin films at different excitation wavelengths at RT. The values are CIE $(x ; y)$ color coordinates obtained.
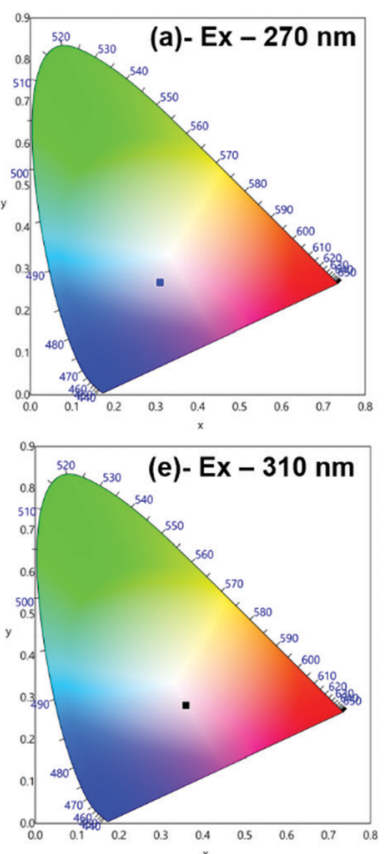
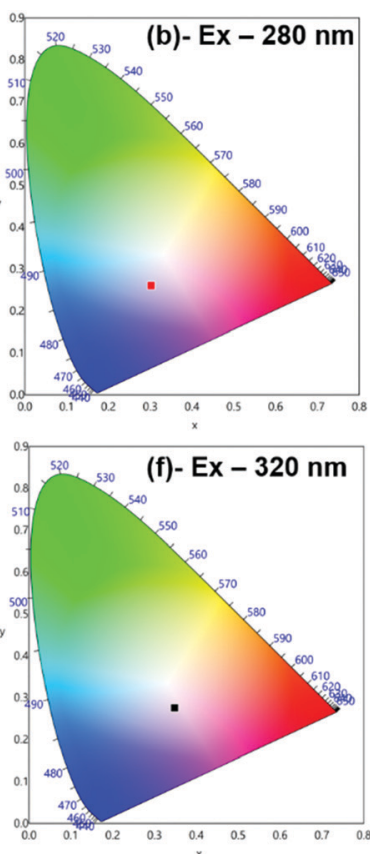
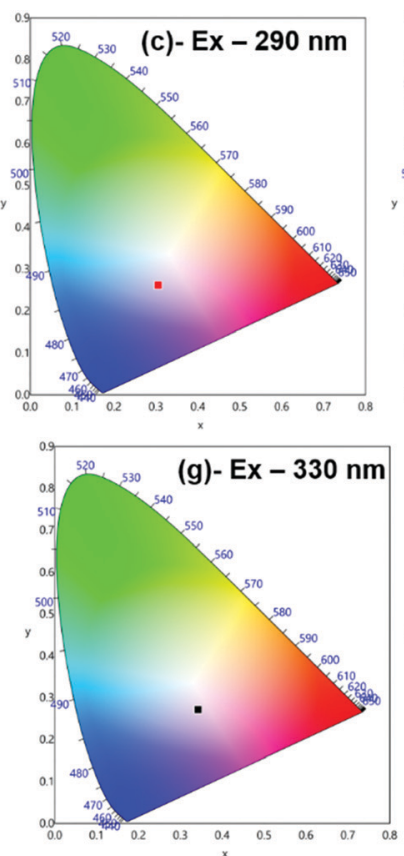

Fig. $111931 \mathrm{CIE}$ Chromaticity diagram of hybrid [Sm(hfaa) $3(\mathrm{Py}-\mathrm{Im})] @ \mathrm{QPU} \_1 \%$ thin film at different excitation wavelength displaying near white-light emission. 
films is in the range of $43.55-54.14 \mu$ s which is almost 4- and 2 -fold shorter than in the solid-state and in DCM solution, this decrease could be attributed to the presence of a high-energy oscillator in the PU polymer matrix, nevertheless it remains at higher end of efficient emitting samarium complexes. To obtain the information for the emitted color by the different hybrid thin films, the CIE color coordinates (Table 2) and diagram were obtained from the steady-state emission spectra and are shown in Fig. 9a and a magnified view of the exact color is also shown in Fig. 9b. As can be seen and as per our assumption from Fig. 9, the developed material displayed color-tunable emission from violet to orange as the doping concentration of the complex was increased from $1-6 \%$. The change in the color from violet to orange is due to the decrease in the $I_{\mathrm{RFL}}$ and can be clearly seen in Fig. S25-S28, ESI. $\dagger$

Moreover, to see more color tunability and to increase the chance to get the near-white light or white light emission from the developed hybrid thin films, the excitation wavelength was varied in the range of $270-340 \mathrm{~nm}$ at intervals of $10 \mathrm{~nm} .1 \%$ and $6 \%$ doped hybrid materials were taken as reference models, as shown in Fig. 10, the steady-state emission spectra of both thin films exhibit increased emission intensity of the ${ }^{4} \mathrm{G}_{5 / 2} \rightarrow{ }^{6} \mathrm{H}_{9 / 2}$ emission transition with $I_{\mathrm{RFL}}$ decreasing simultaneously. The corresponding CIE color coordinates is shown in their respective diagram while CIE chromaticity are shown in Fig. 11 and Fig. S29, ESI. $\dagger$ It can be seen from Fig. S29, ESI, $\dagger$ that $6 \%$ hybrid thin film displayed orange to light pink. Interestingly, the emission of $1 \%$ doped hybrid thin film falls mostly in the white region 1931 CIE chromaticity diagram as can be seen clearly in Fig. 11 . On excitation at 300 and $310 \mathrm{~nm}$, the 1\% doped hybrid thin film exhibited a near white light emission with CIE coordinates of $x=0.3673 ; y=0.2843$ and $x=0.3595 ; y=0.2814$, respectively, which are very close to that for pure white light $(x=0.33, y=0.33)$ according to the $1931 \mathrm{CIE}$ diagram. Therefore this material could be a potential candidate to be used as a single-component material to generate white light or nearwhite light emission for SSL application. ${ }^{34}$

\section{Conclusion}

We have synthesized a medium-dependent color-tunable Sm(III) complex that dimerizes in the solid-state through $\mathrm{N}-\mathrm{H} \cdots \mathrm{O}$ $(2.127 \AA)$ and $\mathrm{N}-\mathrm{H} \cdots \mathrm{F}(2.576 \AA)$ hydrogen bonds. White light emission was achieved by adjustment of doping concentration into a PU polymer and excitation wavelength (as shown in Fig. 10a and 11). $\Delta E$ value of $\approx 7400 \mathrm{~cm}^{-1}$ between the emitting ${ }^{4} \mathrm{G}_{5 / 2}$ level and the next lower energy level in the complex results in effective quenching by high energy oscillators and reduces the overall ET efficiency. This leads to RFL in the range between 350-500 nm, which plays a vital role in obtaining white light emission. The present work opens the door to exploit similar color-tunable Sm(III) based white light materials for the fabrication of full-color display devices and SSL applications.

\section{Conflicts of interest}

There are no conflicts of interest to declare in this work.

\section{Acknowledgements}

MSK acknowledges His Majesty's Trust Fund for Strategic Research (Grant No. SR/SQU/SCI/CHEM/16/02) for funding. RI thanks the HM's Trust Fund for a post-doctoral fellowship. The authors acknowledge the Faculty of Arts and Sciences, Ondokuz Mayis University, Turkey, for the use of the Stoe IPDS 2 diffractometer (purchased under grant F.279 of the University Research Fund). PRR is grateful to the Engineering and Physical Sciences Research Council (EPSRC) for continued support (Grant EP/K004956/1).

\section{References}

1 (a) R. Ilmi, M. S. Khan, Z. Li, L. Zhou, W.-Y. Wong, F. Marken and P. R. Raithby, Inorg. Chem., 2019, 58, 8316-8331; (b) Y. He, L. Liu, G. Fu, W. Li, X. Lü, H. He and W.-Y. Wong, J. Mater. Chem. C, 2019, 7, 4800-4807.

2 (a) R. Ilmi, S. Anjum, A. Haque and M. S. Khan, J. Photochem. Photobiol., A, 2019, 383, 111968; (b) P. K. Shahi, A. K. Singh, S. K. Singh, S. B. Rai and B. Ullrich, ACS Appl. Mater. Interfaces, 2015, 7, 18231-18239; (c) R. Ilmi, S. Kansiz, N. Dege and M. S. Khan, J. Photochem. Photobiol., A, 2019, 377, 268-281.

3 (a) R. Cui, W. Liu, L. Zhou, X. Zhao, Y. Jiang, Y. Zheng and H. Zhang, J. Mater. Chem. C, 2017, 5, 2066-2073; (b) L. Zhou, L. Li, Y. Jiang, R. Cui, Y. Li, X. Zhao and H. Zhang, ACS Appl. Mater. Interfaces, 2015, 7, 16046-16053.

4 (a) K. Yanagisawa, Y. Kitagawa, T. Nakanishi, T. Seki, K. Fushimi, H. Ito and Y. Hasegawa, Chem. - Eur. J., 2018, 24, 1956-1961; (b) C. D. S. Brites, A. Millán and L. D. Carlos, in Handbook on the Physics and Chemistry of Rare Earths, ed. B. Jean-Claude and K. P. Vitalij, Elsevier, 2016, vol. 49, pp. 339-427; (c) D. Errulat, R. Marin, D. A. Gálico, K. L. M. Harriman, A. Pialat, B. Gabidullin, F. Iikawa, O. D. D. Couto, J. O. Moilanen, E. Hemmer, F. A. Sigoli and M. Murugesu, ACS Cent. Sci., 2019, 5, 1187-1198.

5 (a) J. A. Smith, M. A. Singh-Wilmot, K. P. Carter, C. L. Cahill and J. A. Ridenour, Cryst. Growth Des., 2018, 19, 305-319; (b) J.-H. Qin, H.-R. Wang, M.-L. Han, X.-H. Chang and L.-F. Ma, Dalton Trans., 2017, 46, 15434-15442.

6 Y. Hasegawa, S.-i. Tsuruoka, T. Yoshida, H. Kawai and T. Kawai, J. Phys. Chem. A, 2008, 112, 803-807.

7 (a) Y.-H. Zhang, X. Li and S. Song, Chem. Commun., 2013, 49, 10397-10399; (b) Q.-Y. Yang, K. Wu, J.-J. Jiang, C.-W. Hsu, M. Pan, J.-M. Lehn and C.-Y. Su, Chem. Commun., 2014, 50, 7702-7704; (c) J. Zhang, H. Li, P. Chen, W. Sun, T. Gao and P. Yan, J. Mater. Chem. C, 2015, 3, 1799-1806; (d) Y. Xiao, S.-H. Wang, F.-K. Zheng, M.-F. Wu, J. Xu, Z.-F. Liu, J. Chen, R. Li and G.-C. Guo, CrystEngComm, 2016, 18, 721-727; (e) J. Leng, H. Li, P. Chen, W. Sun, T. Gao and P. Yan, Dalton Trans., 2014, 43, 12228-12235.

8 (a) H. Kawai, C. Zhao, S.-I. Tsuruoka, T. Yoshida, Y. Hasegawa and T. Kawai, J. Alloys Compd., 2009, 488, 612-614; (b) T. Harada, K. Tokuda and K. Nishiyama, J. Mol. Liq., 2014, 200, 77-80; (c) K. Miyata, T. Nakagawa, R. Kawakami, Y. Kita, K. Sugimoto, 
T. Nakashima, T. Harada, T. Kawai and Y. Hasegawa, Chem. Eur. J., 2011, 17, 521-528.

9 (a) D. Parker and J. A. G. Williams, J. Chem. Soc., Dalton Trans., 1996, 3613-3628, DOI: 10.1039/DT9960003613; (b) Y. Hasegawa, T. Ohkubo, K. Sogabe, Y. Kawamura, Y. Wada, N. Nakashima and S. Yanagida, Angew. Chem., Int. Ed., 2000, 39, 357-360.

10 K. Yanagisawa, T. Nakanishi, Y. Kitagawa, T. Seki, T. Akama, M. Kobayashi, T. Taketsugu, H. Ito, K. Fushimi and Y. Hasegawa, Eur. J. Inorg. Chem., 2015, 4769-4774.

11 (a) K. Binnemans, K. Lodewyckx, T. Cardinaels, T. N. ParacVogt, C. Bourgogne, D. Guillon and B. Donnio, Eur. J. Inorg. Chem., 2006, 150-157; (b) T. K. Ronson, H. Adams, L. P. Harding, S. J. A. Pope, D. Sykes, S. Faulkner and M. D. Ward, Dalton Trans., 2007, 1006-1022, DOI: 10.1039/B618258E.

12 D. B. G. Williams and M. Lawton, J. Org. Chem., 2010, 75, 8351-8354.

13 G. Sheldrick, Acta Crystallogr., Sect. A: Found. Adv., 2015, 71, 3-8. 14 G. Sheldrick, Acta Crystallogr., Sect. C: Struct. Chem., 2015, 71, 3-8. 15 C. F. Macrae, P. R. Edgington, P. McCabe, E. Pidcock, G. P. Shields, R. Taylor, M. Towler and J. van de Streek, J. Appl. Crystallogr., 2006, 39, 453-457.

16 A. Spek, J. Appl. Crystallogr., 2003, 36, 7-13.

17 L. Farrugia, J. Appl. Crystallogr., 1999, 32, 837-838.

18 J. J. McKinnon, D. Jayatilaka and M. A. Spackman, Chem. Commun., 2007, 3814, DOI: 10.1039/b704980c.

19 M. A. Spackman and D. Jayatilaka, CrystEngComm, 2009, 11, 19-32.

20 M. J. Turner, J. J. McKinnon, S. K. Wolff, D. J. Grimwood, P. R. Spackman, D. Jayatilaka and M. A. Spackman, CrystalExplorer17, The University of Western Australia, Perth, WA, Australia, 2017.

21 (a) D. R. van Staveren, G. A. van Albada, J. G. Haasnoot, H. Kooijman, A. M. M. Lanfredi, P. J. Nieuwenhuizen, A. L. Spek, F. Ugozzoli, T. Weyhermüller and J. Reedijk, Inorg. Chim. Acta, 2001, 315, 163-171; (b) W. J. Evans, D. G. Giarikos, M. A. Johnston, M. A. Greci and J. W. Ziller, J. Chem. Soc., Dalton Trans., 2002, 520-526, DOI: 10.1039/B104095M.
22 (a) Y. Oueslati, S. Kansız, A. Valkonen, T. Sahbani, N. Dege and W. Smirani, J. Mol. Struct., 2019, 1196, 499-507; (b) S. Kansız, A. Tolan, H. İçbudak and N. Dege, J. Mol. Struct., 2019, 1190, 102-115; (c) W. Guerrab, I.-M. Chung, S. Kansiz, J. T. Mague, N. Dege, J. Taoufik, R. Salghi, I. H. Ali, M. I. Khan, H. Lgaz and Y. Ramli, J. Mol. Struct., 2019, 1197, 369-376.

23 (a) G. Stein and E. Würzberg, J. Chem. Phys., 1975, 62, 208-213; (b) M. Latva, H. Takalo, V. M. Mukkala, C. Matachescu, J. C. RodriguezUbis and J. Kankare, J. Lumin., 1997, 75, 149-169; (c) C.-L. Ho, H. Li and W.-Y. Wong, J. Organomet. Chem., 2014, 751, 261-285.

24 S. V. Eliseeva, M. Ryazanov, F. Gumy, S. I. Troyanov, L. S. Lepnev, J.-C. G. Bünzli and N. P. Kuzmina, Eur. J. Inorg. Chem., 2006, 4809-4820.

25 Y. Zheng, L. Fu, Y. Zhou, J. Yu, Y. Yu, S. Wang and H. Zhang, J. Mater. Chem., 2002, 12, 919-923.

26 M. D. Regulacio, M. H. Pablico, J. A. Vasquez, P. N. Myers, S. Gentry, M. Prushan, S.-W. Tam-Chang and S. L. Stoll, Inorg. Chem., 2008, 47, 1512-1523.

27 R. Ilmi and K. Iftikhar, Polyhedron, 2017, 127, 191-202.

28 J. Shi, Y. Hou, W. Chu, X. Shi, H. Gu, B. Wang and Z. Sun, Inorg. Chem., 2013, 52, 5013-5022.

29 Z. Li, J. Yu, L. Zhou, H. Zhang and R. Deng, Inorg. Chem. Commun., 2008, 11, 1284-1287.

30 W. T. Carnall, P. R. Fields and K. Rajnak, J. Chem. Phys., 1968, 49, 4424-4442.

31 A. Døssing, Eur. J. Inorg. Chem., 2005, 1425-1434.

32 W.-S. Lo, J. Zhang, W.-T. Wong and G.-L. Law, Inorg. Chem., 2015, 54, 3725-3727.

33 K. Binnemans, Chem. Rev., 2009, 109, 4283-4374.

34 (a) R. Ilmi, M. S. Khan, W. Sun, L. Zhou, W.-Y. R. Wong and P. R. Raithby, J. Mater. Chem. C, 2019, 7, 13966-13975; (b) L. Ying, C.-L. Ho, H. Wu, Y. Cao and W.-Y. Wong, Adv. Mater., 2014, 26, 2459-2473; (c) X. Yang, G. Zhou and W.-Y. Wong, J. Mater. Chem. C, 2014, 2, 1760-1778; (d) G. Zhou, W.-Y. Wong and S. Suo, J. Photochem. Photobiol., C, 2010, 11, 133-156. 